\title{
Ultrasensitive Anti-Interference Voice Recognition by Bio-Inspired Skin-Attachable Self-Cleaning Acoustic Sensors
}

Truong-Son Dinh Le, ${ }^{\dagger, *}, \#$ Jianing An, ${ }^{\dagger, \#, *}$ Yi Huang,,$^{\dagger}$ Quoc Vo, ${ }^{\dagger}$ Jeeranan Boonruangkan, ${ }^{\dagger}$ Tuan Tran, ${ }^{\dagger}$ Seung-Woo Kim,,${ }^{\S}$ Gengzhi Sun, $,{ }^{\perp}, *$ and Young-Jin Kim ${ }^{\dagger}, \S, *$

†School of Mechanical and Aerospace Engineering, Nanyang Technological University (NTU), 50 Nanyang Avenue, 639798, Singapore

†Singapore Centre for 3D printing, School of Mechanical and Aerospace Engineering, Nanyang Technological University (NTU), 50 Nanyang Avenue, 639798, Singapore

§Department of Mechanical Engineering, Korea Advanced Institute of Science and Technology (KAIST), Science Town, Daejeon, 34141, South Korea

"Institute of Advanced Materials (IAM), Nanjing Tech University (NanjingTech), 30 South Puzhu Road, Nanjing, 211816, P.R. China

${ }^{\perp}$ Institute of Flexible Electronics (IFE), Northwestern Polytechnical University, 127 West Youyi Road, Xi'an 710072, P. R. China

*Corresponding authors: anjianing@ntu.edu.sg, iamgzsun@njtech.edu.cn, yj.kim@kaist.ac.kr 


\section{This PDF file includes:}

Figure S1. EDS characterization of the film produced at writing speed of $10 \mathrm{~mm} \mathrm{~s}^{-1}$.

Figure S2. High-resolution C1s, O1s, and Si2p XPS spectra of the patterned composite film and the pristine PDMS.

Figure S3. Characterizations of pristine GO and laser-patterned films at different writing speeds.

Figure S4. Surface wettability of pristine GO film.

Figure S5. Surface roughness of pristine GO and laser-patterned films at different writing speeds.

Figure S6. Characterizations of the film written with the speed of $1 \mathrm{~mm} \mathrm{~s}^{-1}$.

Figure S7. Correlation between bending strain, bending radius, and thickness of the sensor subjected to a bending moment.

Figure S8. SEM image showing generation of microcrack arrays into the rGO/PDMS composite film.

Figure S9. Water-proof capability of the rGO/PDMS composite strain sensor.

Figure S10. Optical images showing the experimental setup to examine the electromechanical properties of the sensor.

Figure S11. Digital photographs of the microcracks under different bending strains.

Figure S12. $I-V$ curves characteristics of the strain sensor.

Figure S13. Photographs of sensors attached on the piezoelectric benders to measure minute strain.

Figure S14. Monitoring pulse signal using the strain sensor.

Figure S15. SEM images and electromechanical characteristics of the laser-patterned films produced at different writing speeds after being bent around a metal rod.

Figure S16. Hysteresis performance of the sensor at different strains.

Figure S17. Cross-sectional SEM image of rGO/PDMS composite film.

Figure S18. Resistance changes and SEM images of the rGO/PDMS composite film before and after tape peeling test.

Figure S19. Temporal (top) and frequency (bottom) responses of the strain sensor at excitation frequencies at 1000 and $5000 \mathrm{~Hz}$, in comparison with those of a commercial strain gauge.

Figure S20. Response of the strain sensor to sound waves emitted by a loudspeaker.

Figure S21. Acoustic signal waveforms and spectrograms of the phonation of "NTU" by speaker female 2 and male 2 .

Figure S22. Schematic of the FsLDW platform. 
Table S1. A comparison of anti-interference performance among different skin-attached acoustic sensors.

Captions for Movies S1 to S8

References

Other Supporting Materials for this manuscript include the following:

Movies S1 to S8 


\section{Characterizations of photothermal decomposition of PDMS by FsLDW}

The film patterned at $10 \mathrm{~mm} \mathrm{~s}^{-1}$ was characterized using EDS (Figure S1) and XPS (Figure S2). The EDS element mapping (Figure S1a) and corresponding spectrum (Figure S1b) clearly show the presence of $\mathrm{Si}$, indicating the photothermal decomposition of PDMS. Figure S2 compares the deconvolution of $\mathrm{C} 1 \mathrm{~s}, \mathrm{O} 1 \mathrm{~s}$ and $\mathrm{Si} 2 \mathrm{p}$ peaks for pristine PDMS and the patterned film. Notably, the O-Si-O bonds with the correspondent O1s peak at $533.1 \mathrm{eV}$ in the $\mathrm{O} 1 \mathrm{~s}$ spectrum and the correspondent Si2p peak at $103.4 \mathrm{eV}$ in the Si2p spectrum are more prominent for the patterned film compared with pristine PDMS. This can be attributed to the bonding of remaining silicon with atmospheric oxygen, due to the splitting of Si-C and the carbon loss induced by photothermal decomposition of PDMS. ${ }^{1}$
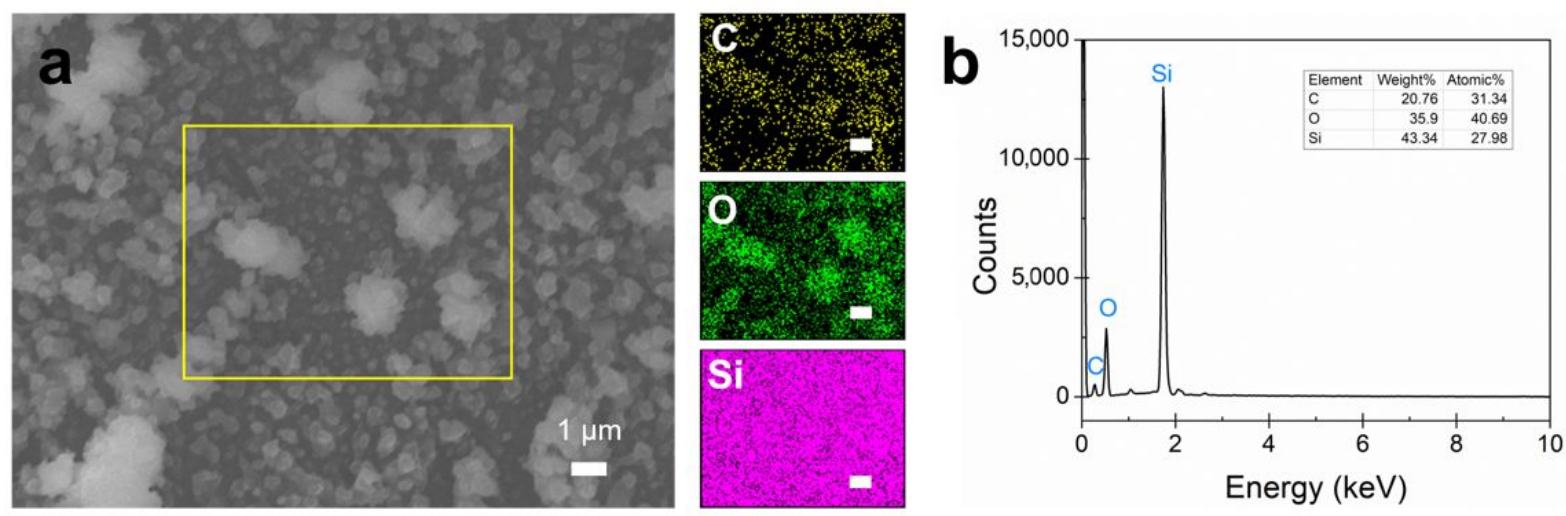

Figure S1. EDS characterization of the film produced at writing speed of $10 \mathrm{~mm} \mathrm{~s}^{-1}$. (a) The element $(\mathrm{C}, \mathrm{O}$, and $\mathrm{Si}$ ) mapping, scale bar in the element mapping images is $1 \mu \mathrm{m}$. (b) The spectrum showing the element composition.

C1s

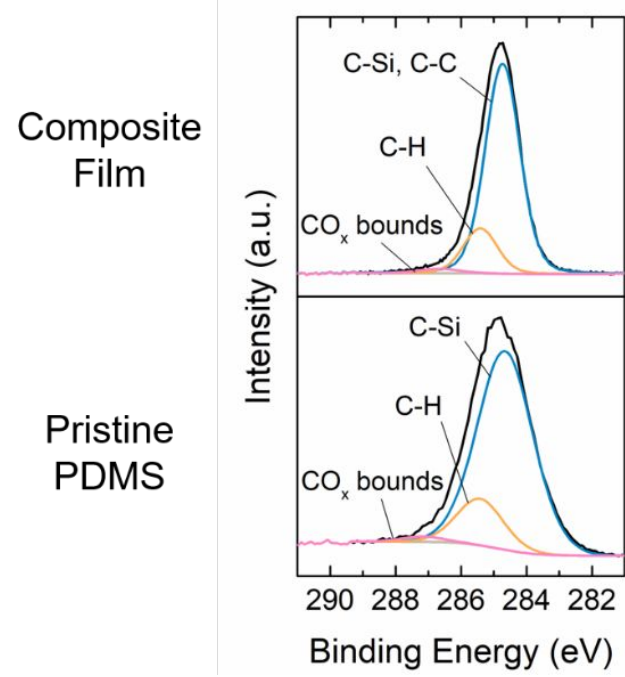

O1s

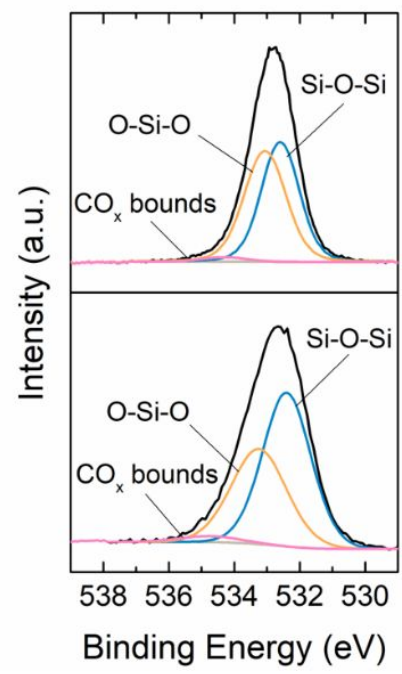

$\mathrm{Si} 2 \mathrm{p}$

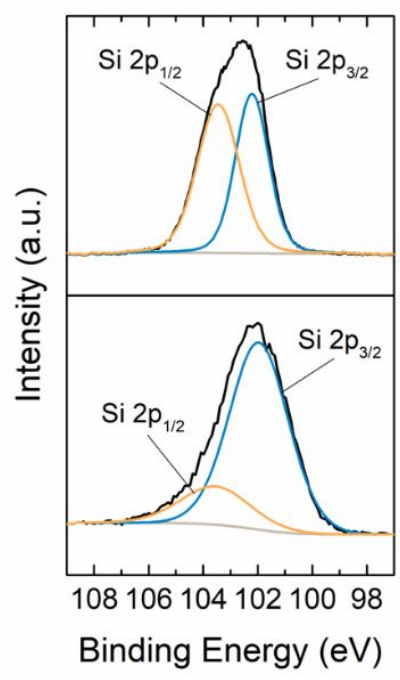

Figure S2. High-resolution C1s, O1s, and Si2p XPS spectra of the patterned composite film and the pristine PDMS. 

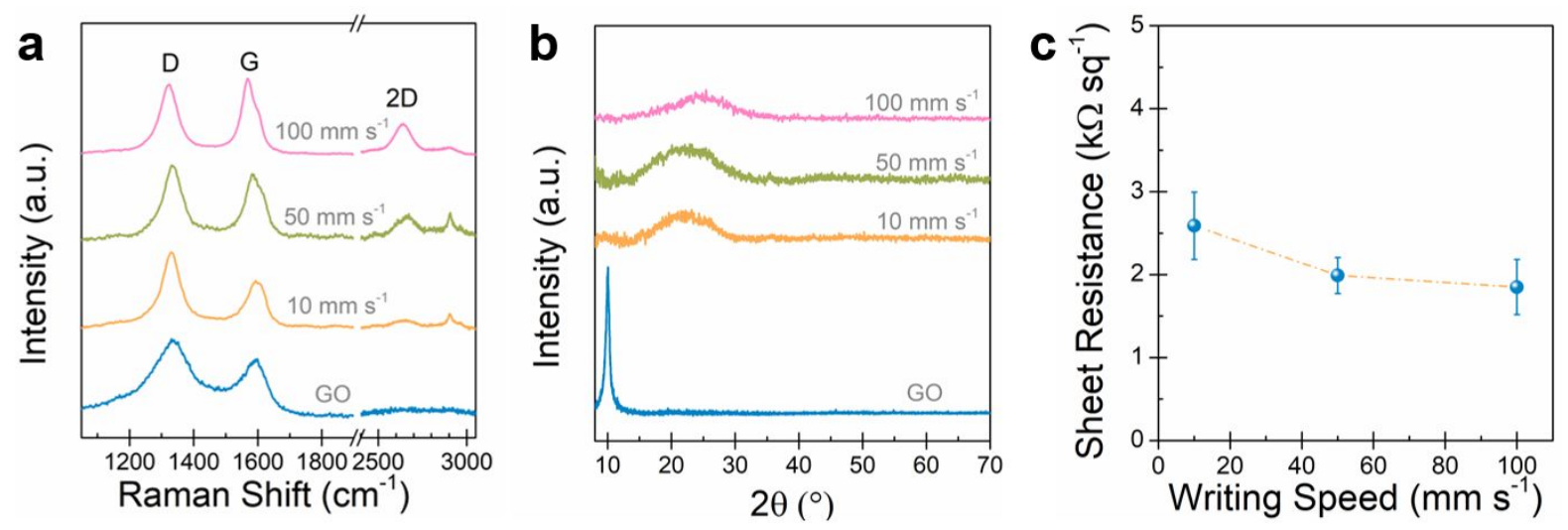

Figure S3. Characterizations of pristine GO and laser-patterned films at different writing speeds. (a) Raman spectra and (b) XRD patterns of pristine GO film and laser-patterned films. (c) Plot of sheet resistance as a function of writing speed. The error bars result from re-sampling.



Figure S4. Surface wettability of pristine GO film. (a) SEM image of a GO film. (b) Optical photograph showing the water contact angle of the GO film.
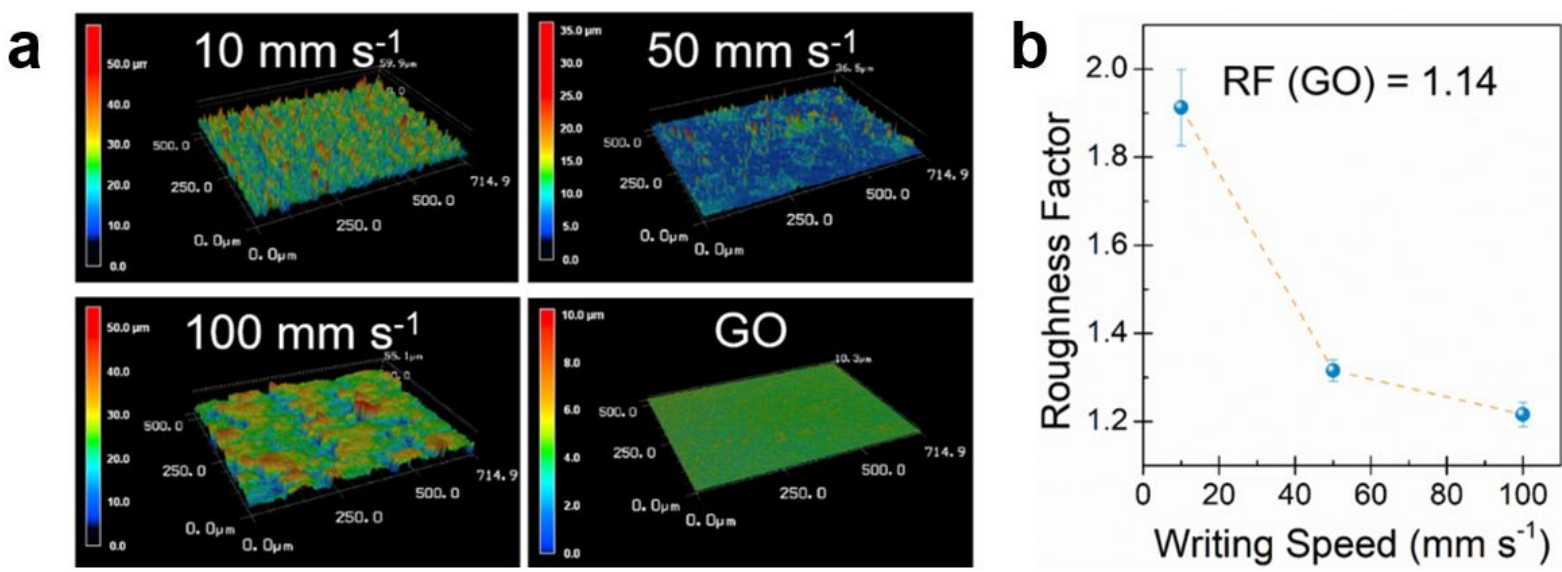

Figure S5. Surface roughness of pristine GO and laser-patterned films at different writing speeds. (a) 3D laser profile images of patterned films at different writing speeds and pristine GO film. (b) Plot of surface roughness factor of patterned films and pristine GO film. 


\section{Characterizations of the film written with the speed of $1 \mathrm{~mm} \mathrm{~s}^{-1}$}

An as-deposited GO film was written with a low speed of $1 \mathrm{~mm} \mathrm{~s}^{-1}$, the surface wettability and conductivity of the sample were subsequently characterized. The SEM image (Figure S6a) shows the aggregation of irregular nanostructures with voids. the corresponding surface profile measured by the 3D laser scanning confocal microscope is shown in Figure S6b. The roughness factor of the surface is 1.57 , which is smaller than that of the surface modified by writing speed of $10 \mathrm{~mm} \mathrm{~s}^{-1}$; as a result, the contact angle is only $142.6^{\circ}$ (Figure S6c). On the other hand, the sheet resistance of the film is as high as $5.32 \mathrm{M} \Omega ~ s q^{-1}$, which may result from the carbon ablation under elongated laser irradiation (Figure S6d). Its Raman spectrum (Figure S6e) shows weak $\mathrm{D}$ and $\mathrm{G}$ bands that almost merge into the background, further validating the carbon ablation.


Figure S6. Characterizations of the film written with the speed of $1 \mathrm{~mm} \mathrm{~s}^{-1}$. SEM image (a) and 3D profile image (b) of the composite film written at $1 \mathrm{~mm} \mathrm{~s}^{-1}$. (c) Photograph showing the water contact angle. (d) EDS spectrum showing the element composition. (e) Raman spectrum of the film.

\section{Crack-generation process}

The crack-generation system in this study is schematically illustrated in Figure S7. A metal roller with a radius $(r)$ of $1.0 \mathrm{~mm}$ is utilized to induce the bending strain. In addition, two identical calibration weights ( $30 \mathrm{~g}$ for each) are used to ensure that the PDMS substrate can conform the roller. The dimensions of the PDMS substrate are $30 \mathrm{~mm} \times 8 \mathrm{~mm} \times 0.3 \mathrm{~mm}$ (length $\times$ width $\times$ thickness). Because the total thickness of the sensor $(h)$ is $0.3 \mathrm{~mm}$, the bending strain on the thin film can be estimated using the formula ${ }^{2}$ below: 


$$
\varepsilon_{1}=\frac{h}{2 r}=\frac{0.3 \mathrm{~mm}}{2 \times 1.0 \mathrm{~mm}}=0.15=15 \%
$$

where, $\varepsilon_{1}$ is the bending strain on the thin film, $h$ is the total thickness of the PDMS substrate and the sensing layer ( $\approx$ PDMS thickness), and $r$ is the bending radius.

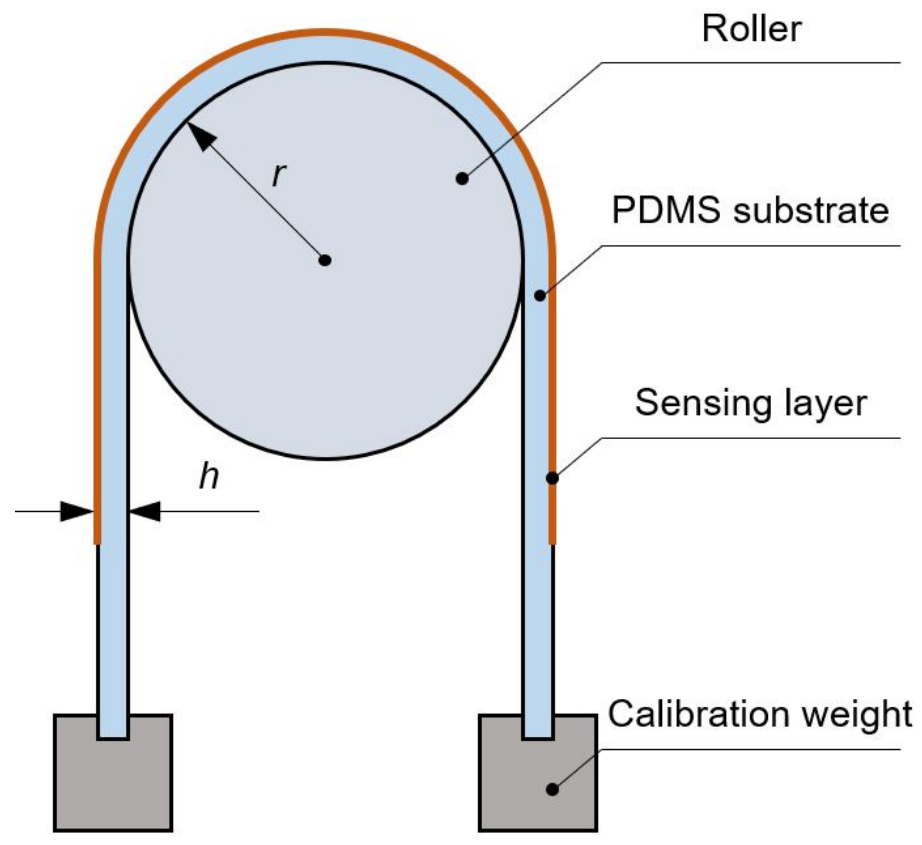

Figure S7. Correlation between bending strain, bending radius, and thickness of the sensor subjected to a bending moment.

The stress on the PDMS substrate induced by the calibration weights:

$$
\text { Stress }=\frac{\text { Force }}{\text { Area }}=\frac{0.03 \mathrm{~kg} \times 9.81 \mathrm{~m} \mathrm{~s}^{-2}}{0.3 \mathrm{~mm} \times 8 \mathrm{~mm}}=122,625 \mathrm{Nm}^{-2}
$$

The stretching strain $\left(\varepsilon_{2}\right)$ is calculated by the formula:

$$
\varepsilon_{2}=\frac{\text { Stress }}{\text { Young's modulus }}=\frac{122,625 \mathrm{Nm}^{-2}}{2.61 \times 10^{6} \mathrm{Nm}^{-2}}=0.047=4.7 \%
$$

Where Young's modulus of PDMS is $2.61 \mathrm{MPa} .^{3}$

Hence, the total strain applied in the thin film is a combination of the bending strain and the stretching strain:

$$
\varepsilon=\varepsilon_{1}+\varepsilon_{2}=15 \%+4.7 \%=19.7 \%
$$

In our study, the microcracks on rGO/PDMS composite film were started to be generated when the film was bent at $3.75 \%$ of strain (corresponding to a bending radius of 4 $\mathrm{mm}$ ); thus, the $19.7 \%$ strain was sufficient to generate the microcracks. At the same time, because the sensing range of our sensor is up to $1.0 \%$, this strain of $19.7 \%$ was high enough to ensure that new microcracks cannot be additionally formed during the operation. These 
experimental results agree well with the previous works reported by Kang and co-workers ${ }^{4}$; they used various radii of curvatures (from 1.0 to $3.0 \mathrm{~mm}$ ) to bend and induce microcracks on a thin platinum film deposited on a poly(urethane acrylate) substrate. They found that smaller bending radius (or higher bending strain) resulted in shorter spacing between the microcracks (or higher crack density) and better sensitivity in strain sensing; the sensitivity was the highest when the microcracks were formed with a bending radius of $1.0 \mathrm{~mm}$ (correspond to $3 \%$ of strain). In our study, whereas, the further increase of the strain (by reducing the bending radius or increasing the weights) started to make damages in the conductive islands due to the elastic deformation and PDMS's Poisson's effect. Considering these, we optimized the total strain to $19.7 \%$ (induced by a $1 \mathrm{~mm}$-roller and two weights) so as to ensure good sensing performance and high stability. In addition, owing to the large rotational inertia of the weights and balancing system, we used a relatively slow rolling speed of $1.0 \mathrm{~mm} \mathrm{~s}^{-1}$ to form the microcracks in a controllable manner."

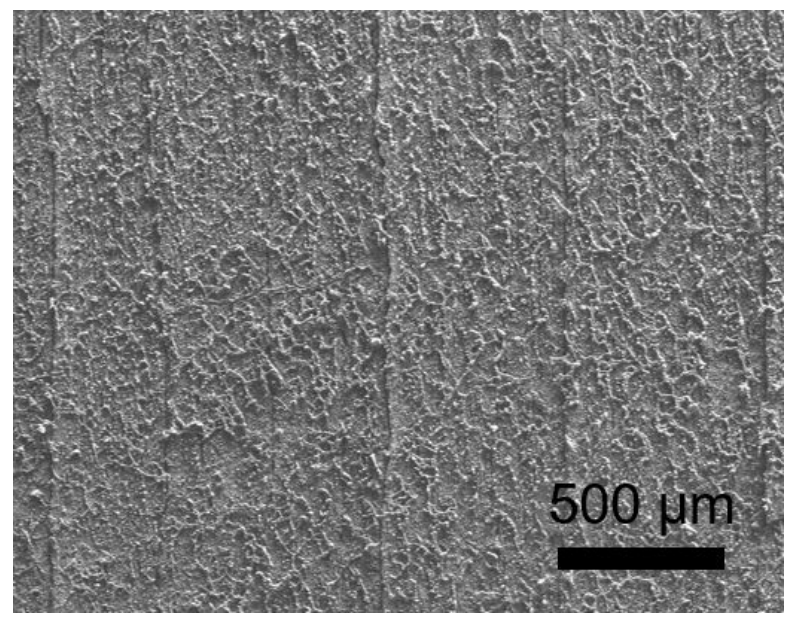

Figure S8. SEM image showing generation of microcrack arrays into the rGO/PDMS composite film.

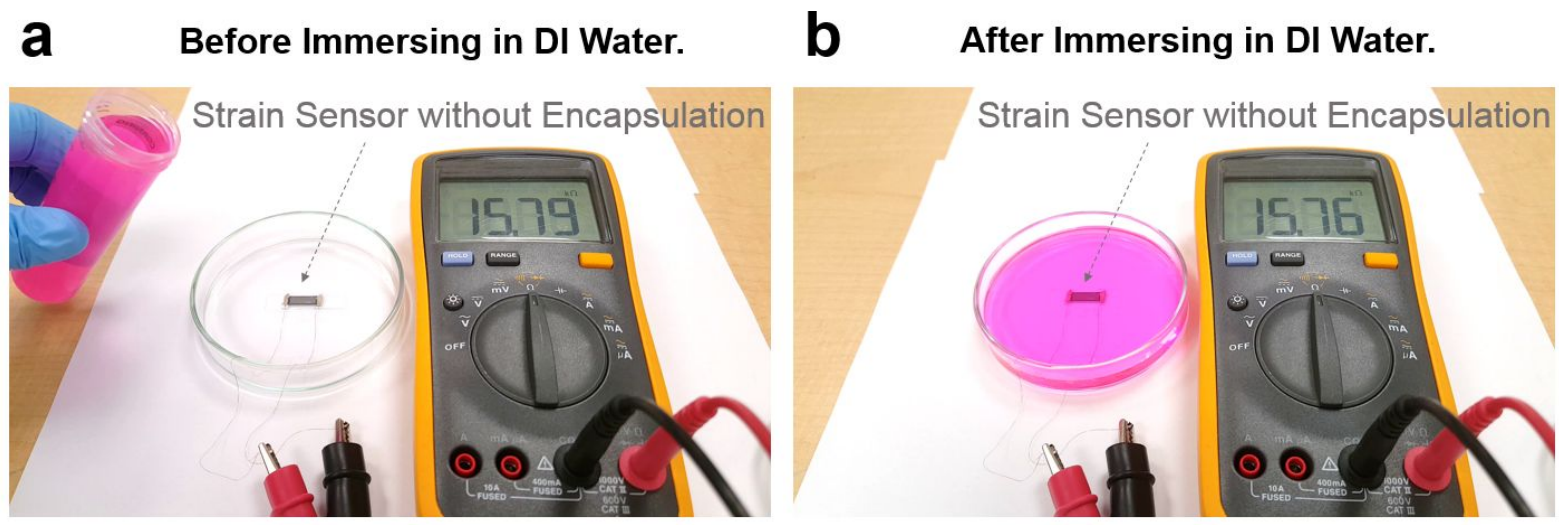

Figure S9. Water-proof capability of the rGO/PDMS composite strain sensor. Snapshots of a strain sensor without encapsulation before (a) and after (b) being immersed in DI water (pigment was added to enhance the contrast). A thin PDMS layer was coated on the silver electrode region to protect the electrode from water-induced electrical influence. The negligible 
relative change in resistance $\left(\Delta R / R_{0}<0.2 \%\right)$ indicates the excellent water-proof property of the strain sensor based on superhydrophobic rGO/PDMS composite film.

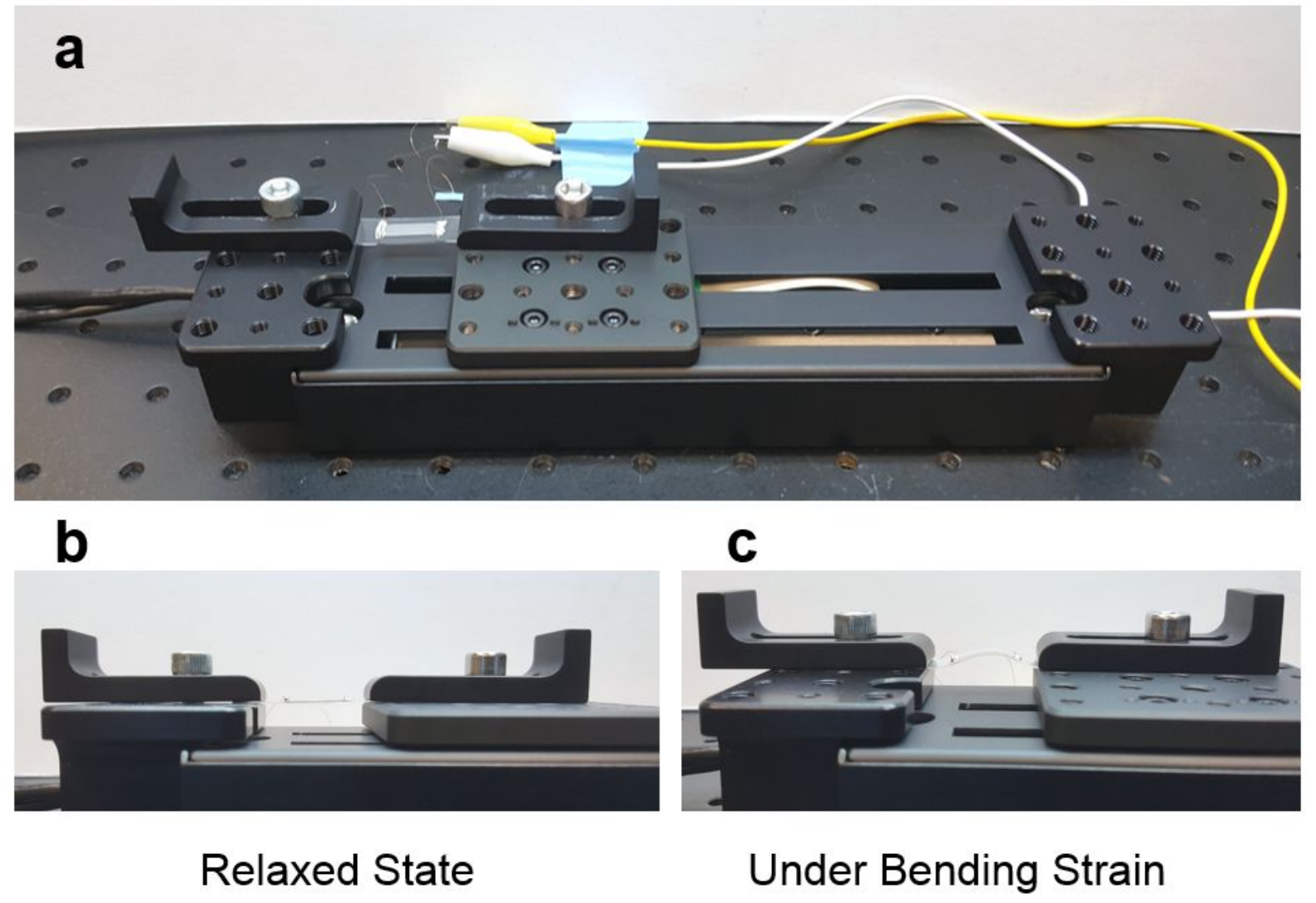

Figure S10. (a) Optical images showing the experimental setup to examine the electromechanical properties of the sensor. (b) The sensor is in relaxed state. (c) The sensor is under bending strain.
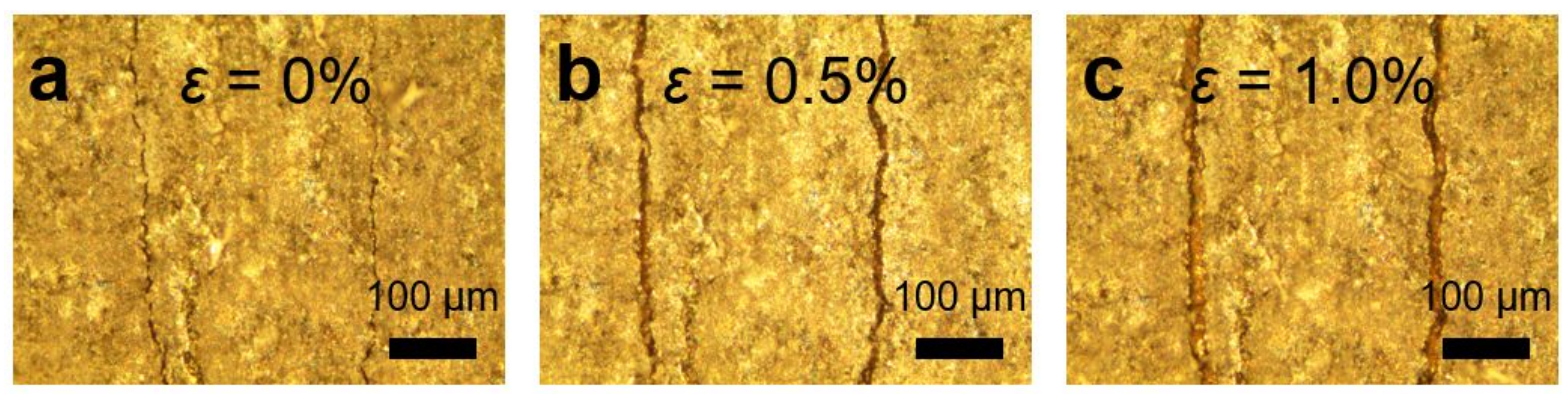

Figure S11. Digital photographs of the microcracks under different bending strains. 

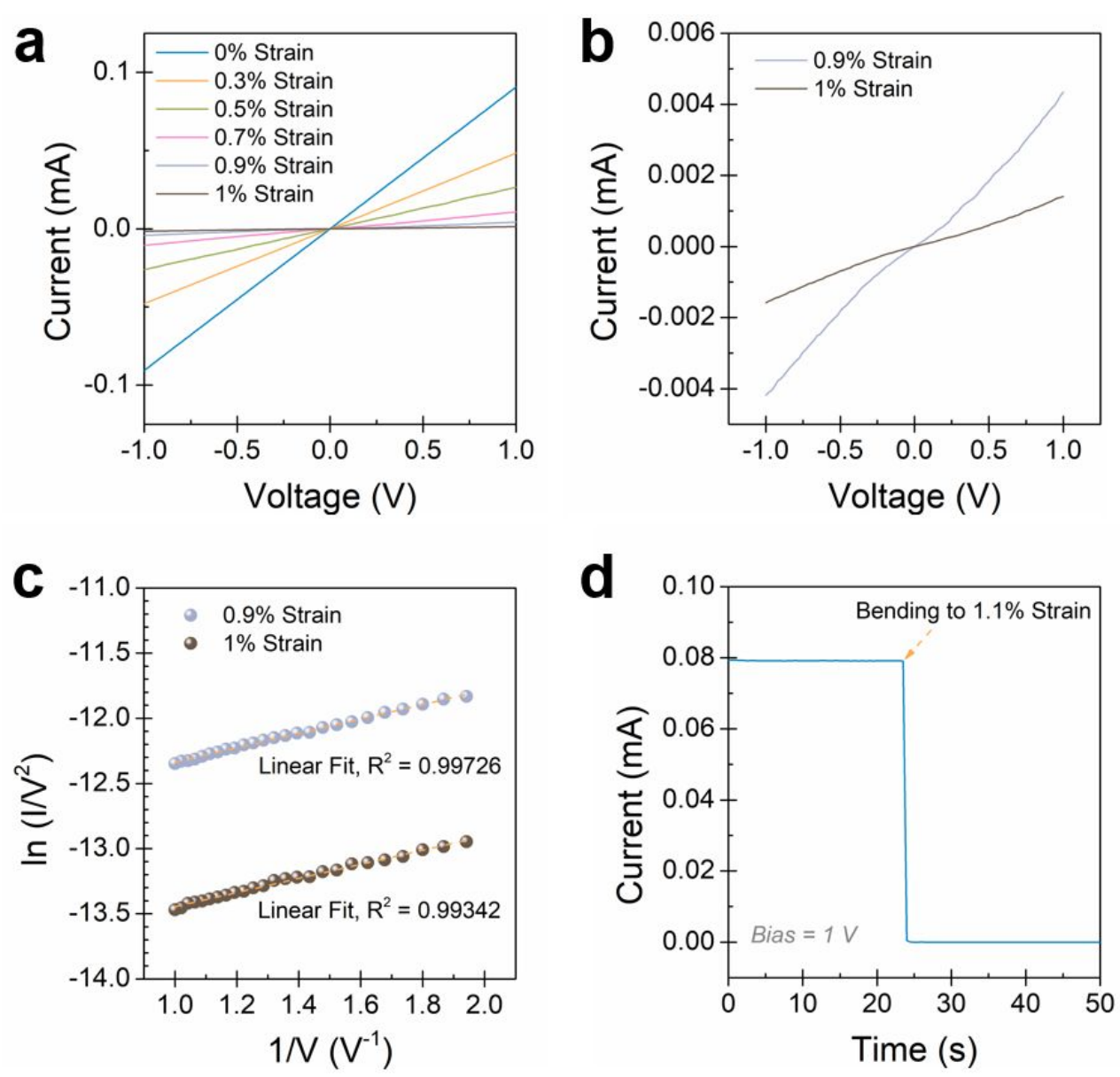

Figure S12. $I-V$ curves characteristics of the strain sensor. (a) $I-V$ curves of the sensor measured at different bending strains. (b) Magnified $I-V$ curves at strains of $0.9 \%$ and $1 \%$. (c) $\ln \left(I / V^{2}\right) v s$. $1 / V$ plots for the sensor at $0.9 \%$ and $1 \%$ bending strains, the plots can be well fitted to Fowler-Nordheim tunnelling. (d) $I-t$ curve showing the drastic resistance increase of the sensor upon bending to $1.1 \%$ strain.
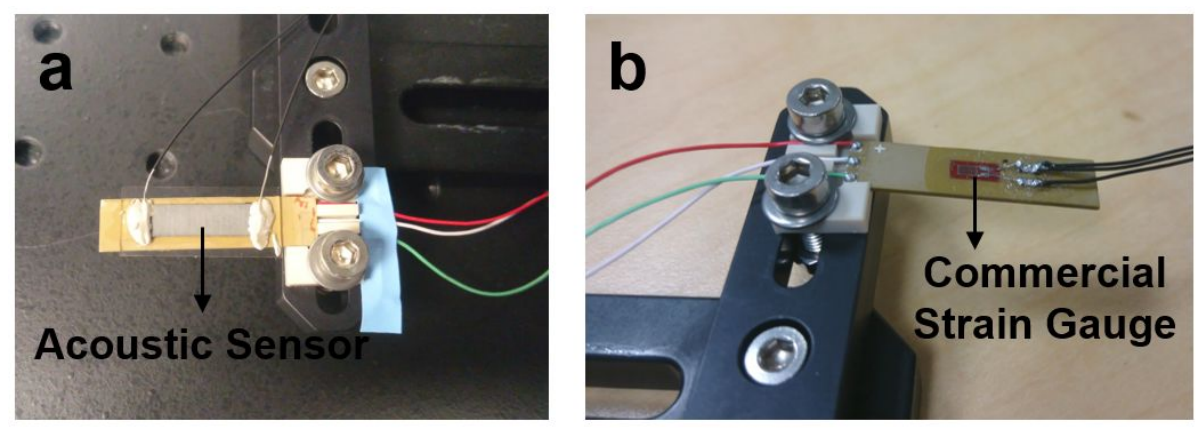

Figure S13. Photographs of sensors attached on the piezoelectric benders to measure minute strain. (a) the acoustic sensor on the piezoelectric bender. (b) the commercial strain gauge on the piezoelectric bender. 

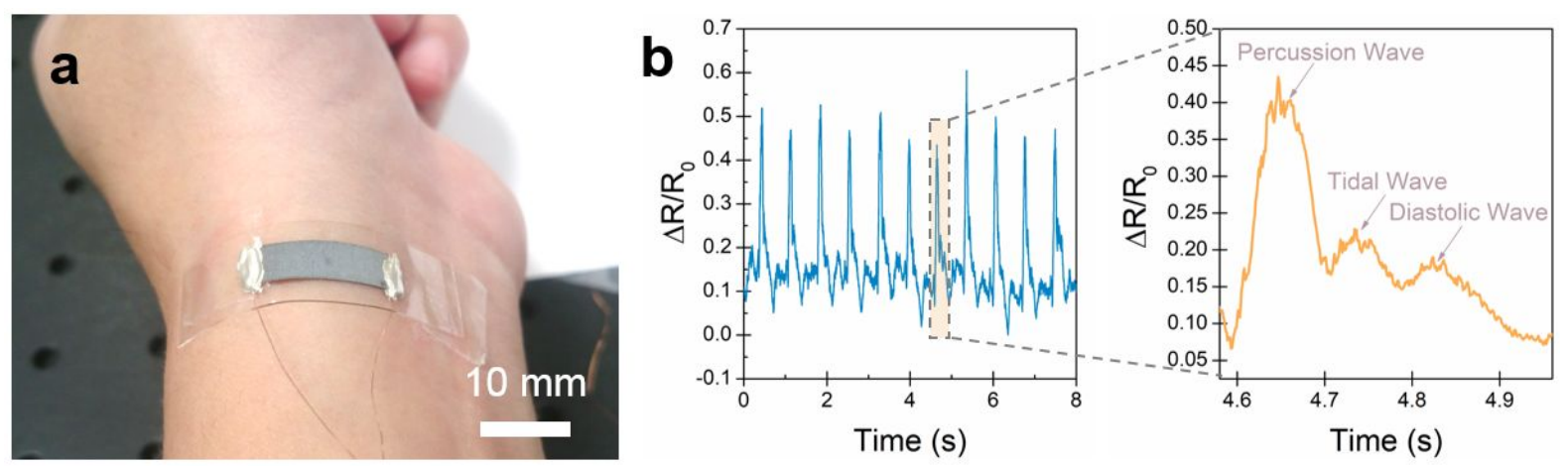

Figure S14. Monitoring pulse signal using the strain sensor. (a) Photograph of the device attached on a human wrist. (b) Measured pulse waveform.
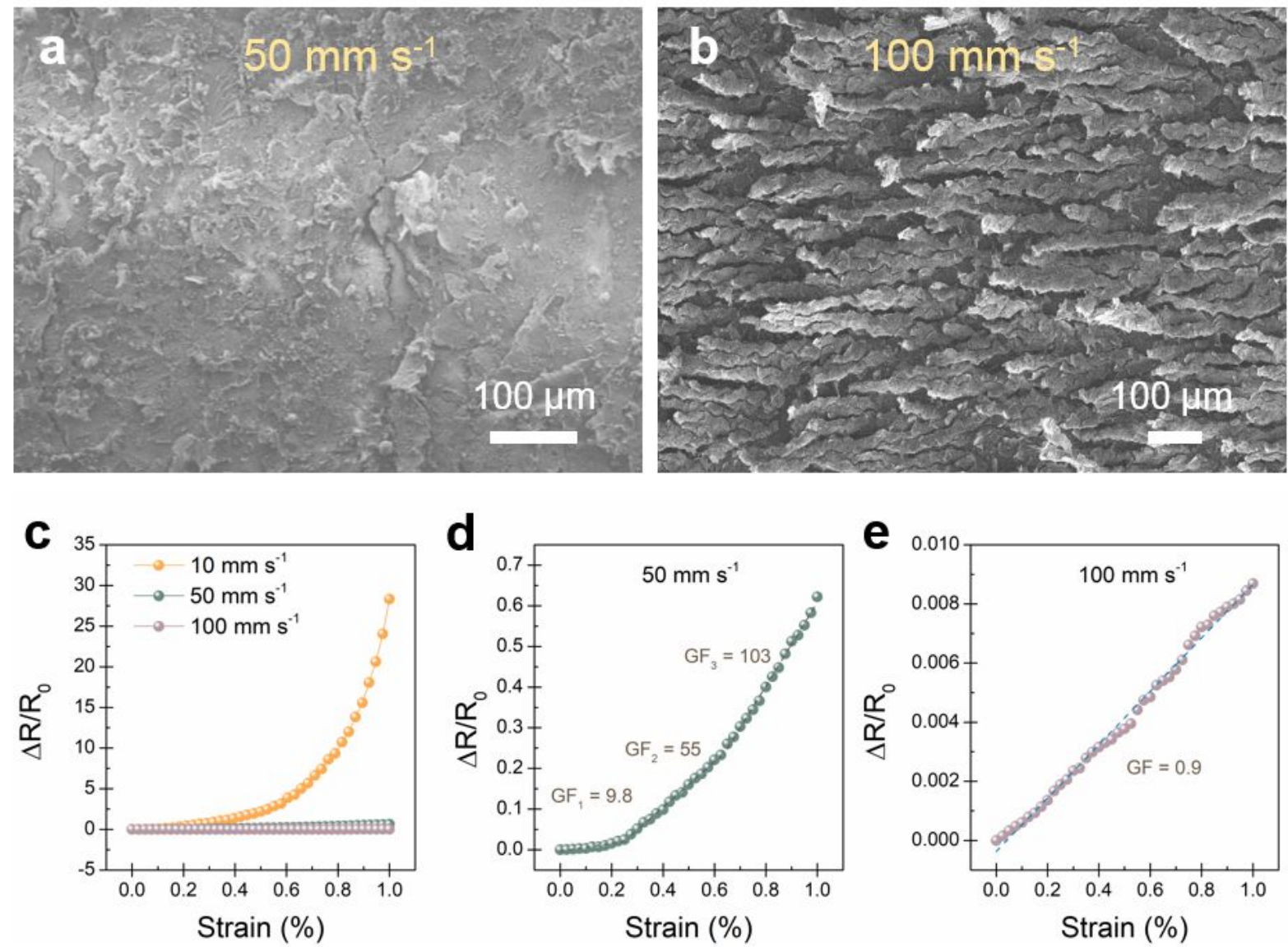

Figure S15. SEM images and electromechanical characteristics of the laser-patterned films produced at different writing speeds after being bent around a metal rod. (a) SEM image of the sampled produced at medium writing speeds. (b) SEM image of the sample produced at high writing speed. (c) Sensitivity comparison of the sensors obtained at the three levels of writing speeds. (d) and (e) Enlarged sensing performances of devices written at speeds of 50 and 100 $\mathrm{mm} \mathrm{s}^{-1}$, respectively. 


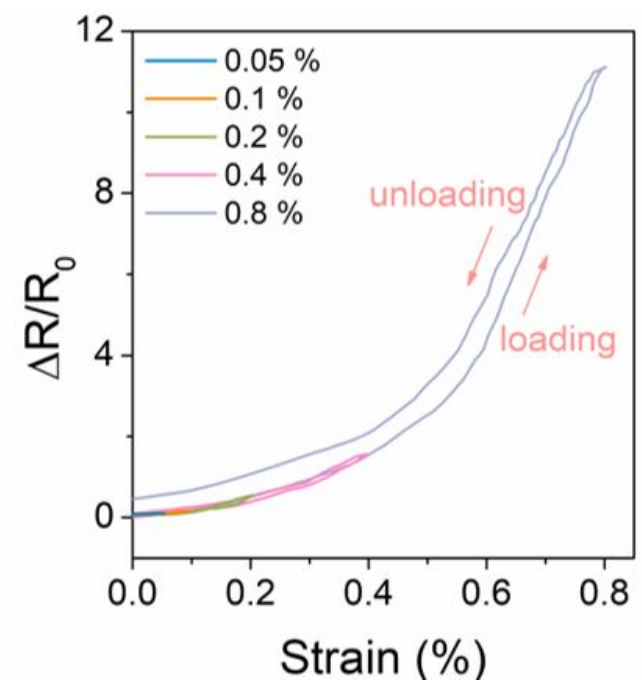

Figure S16. Hysteresis performance of the sensor at different strains.

\section{Mechanical robustness of the strain sensor}

The femtosecond laser irradiation simultaneously enables the photoreduction of GO and photothermal decomposition of PDMS elastomer. As a result, the rGO and molten PDMS are fused together so as to form a hierarchical $\mathrm{rGO} / \mathrm{PDMS}$ composite layer. The cross-sectional SEM image (Figure S17) clearly shows the fused rGO/PDMS composite on the top and the remaining un-melted PDMS layer at the bottom serving as the stretchable substrate. Specifically, the melted PDMS established a strong bonding of the composite film with the substrate, ${ }^{5}$ which endowed the sensor with good mechanical robustness.

In addition, we have conducted the tape peeling tests using a scotch tape. The resistance of the sensor was monitored during the repetitive tape peeling test, as shown in Figure S18a; thirty peel-off cycles only caused a slight increase in resistance $\left(\Delta R / R_{0}<3 \%\right)$. The morphologies of the film before and after tape peeling test were examined by SEM, as shown in Figures S18b and c. There was unnoticeable change in the film morphology induced by the tape peeling, demonstrating its excellent mechanical stability.

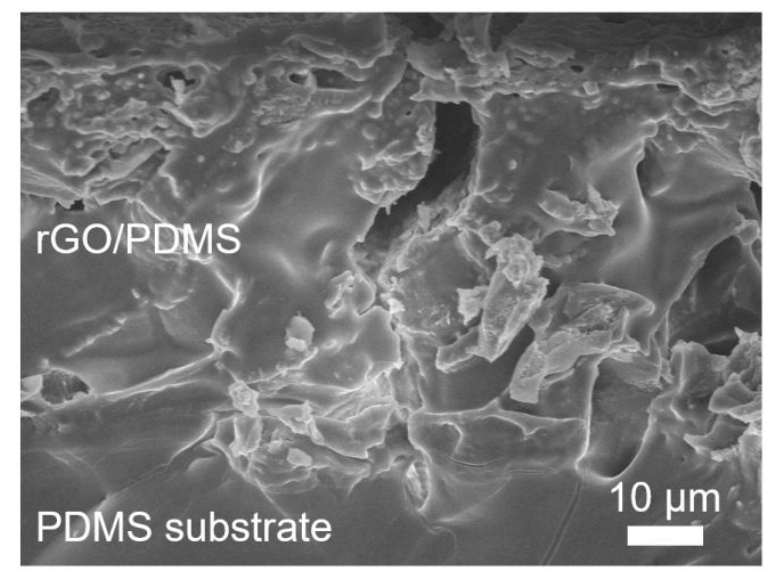

Figure S17. Cross-sectional SEM image of rGO/PDMS composite film. 

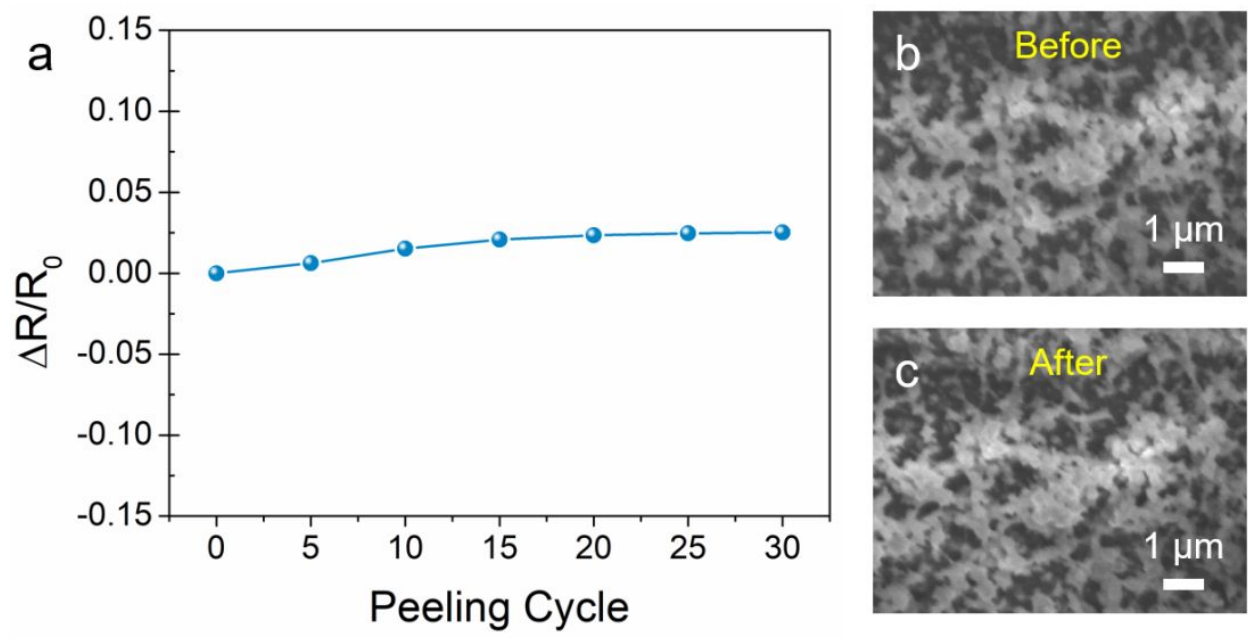

Figure S18. (a) Relative resistance changes as a function of peeling cycle. (b-c) SEM images of the rGO/PDMS composite film before (b) and after (c) repetitive tape peeling test.

$1,000 \mathrm{~Hz}$

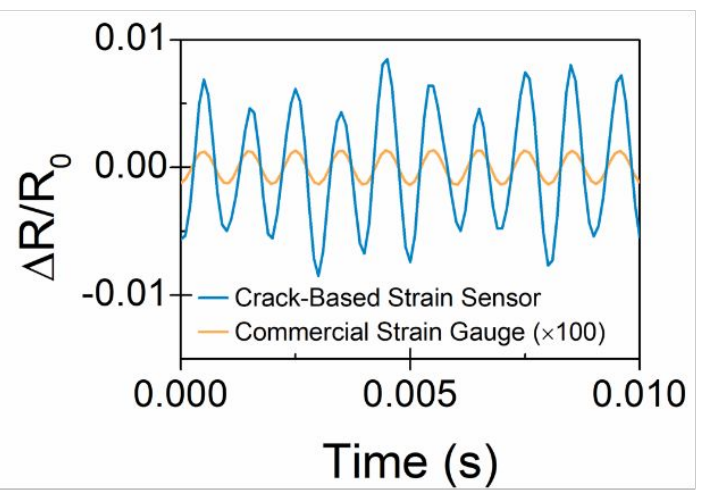

$5,000 \mathrm{~Hz}$

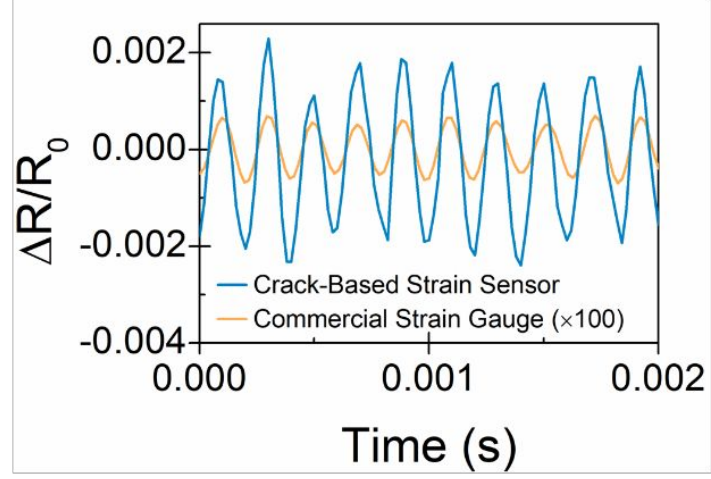

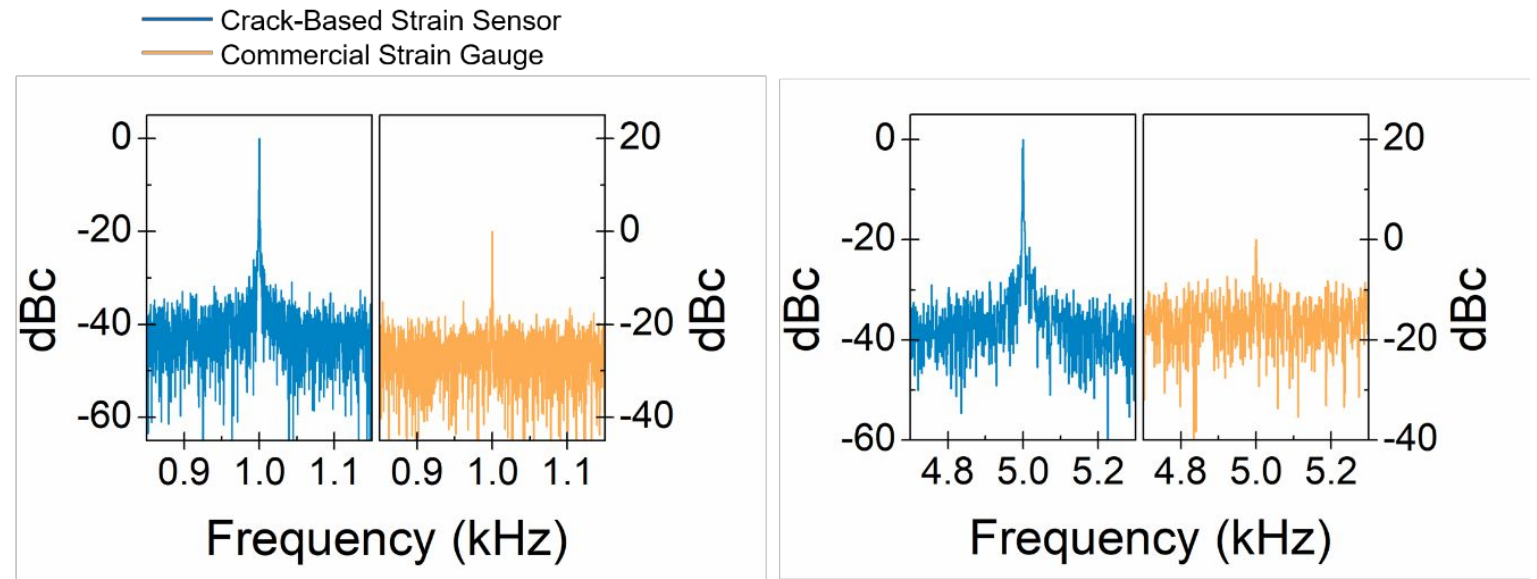

Figure S19. Temporal (top) and frequency (bottom) responses of the strain sensor at excitation frequencies at 1000 and $5000 \mathrm{~Hz}$, in comparison with those of a commercial strain gauge. Commercial strain gauge's signal strengths are displayed with 100 -fold multiplication for the visibility. 


\section{Response of the strain sensor to sound waves emitted by a loudspeaker}

A loudspeaker was employed to emit sound waves over the audible frequency range of 20$20,000 \mathrm{~Hz}$. The acoustic signals generated by the loudspeaker propagated through the air by $1.0 \mathrm{~mm}$ and was detected by our flexible sensor, the resulting voltage variation induced by the resistance change of the sensing film was monitored in real time by an oscilloscope (Figure S20a). Figure S20b presents the frequency response of the sensor with the same input sinusoidal amplitude of $3.5 \mathrm{~V}$. The mechanical resonance frequency was observed at $500 \mathrm{~Hz}$ (in accordance to the resonance frequency of the loudspeaker), and then the response decreases gradually at higher frequencies. The response to vibrational amplitude was also investigated at $500 \mathrm{~Hz}$ as shown in Figure S20c, the voltage increases monotonically with increasing sound pressure level (SPL). Specifically, for testing high-frequency performances, an example time response was recorded at 20,000 Hz - the highest frequency of audible sound (Figure S20d) and its spectrum was analyzed in the frequency domain by taking FFT (Figure S20f). These clearly demonstrate that our crack-based strain sensor can detect and reconstruct the highfrequency sound waves at $20,000 \mathrm{~Hz}$ (corresponding to a response time of $50 \mu \mathrm{s}$ in Figure S20e) with high SNR.
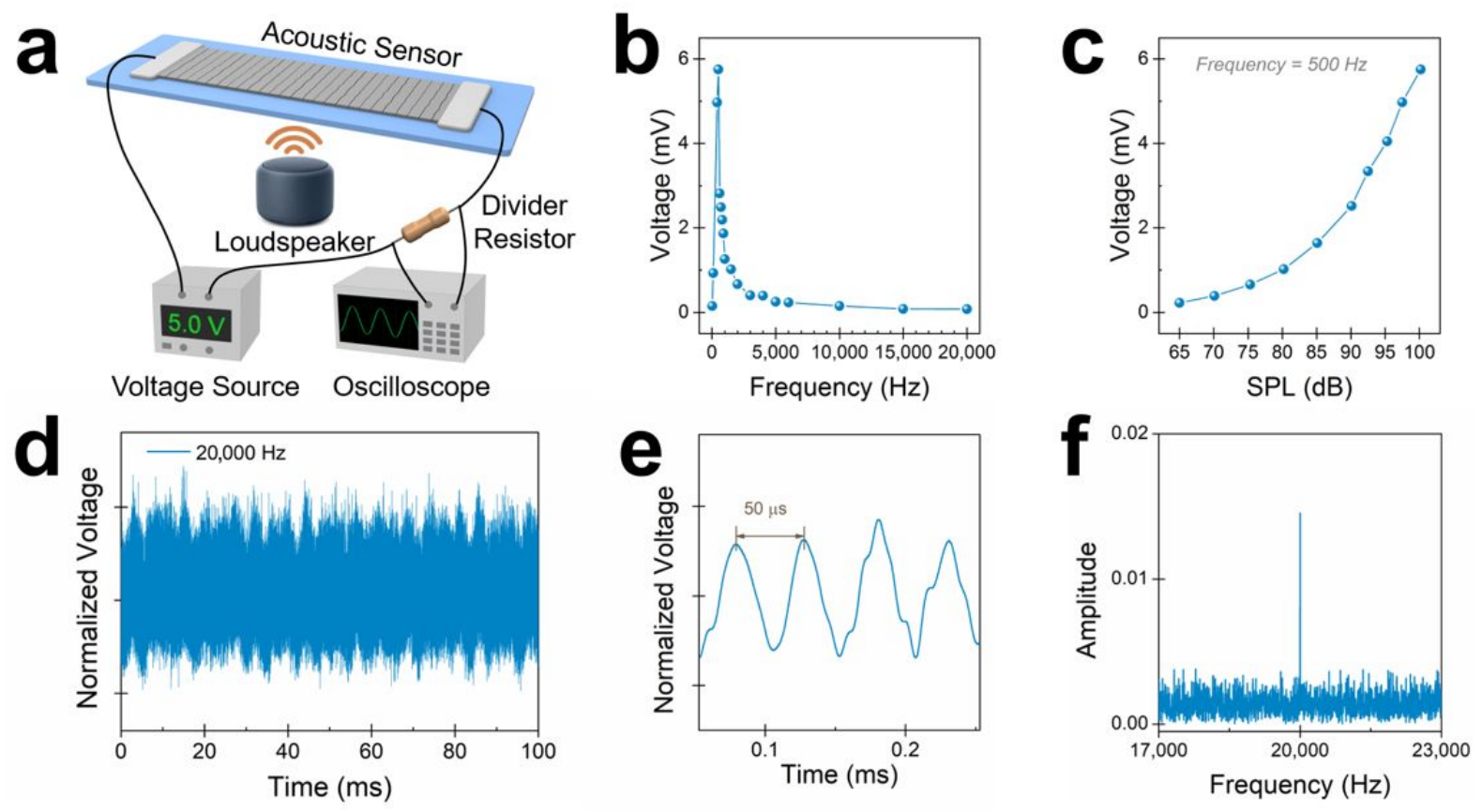

Figure S20. Response of the strain sensor to sound waves emitted by a loudspeaker. (a) Schematic of wide-frequency sound detection experimental setup. (b) The output voltage as a function of sound frequency. (c) The output voltage as a function of SPL at resonance frequency of $500 \mathrm{~Hz}$. (d) Real-time response at frequency of 20,000 Hz. (e) Enlarged view of the waveform. (f) Frequency response to the sound signal. 

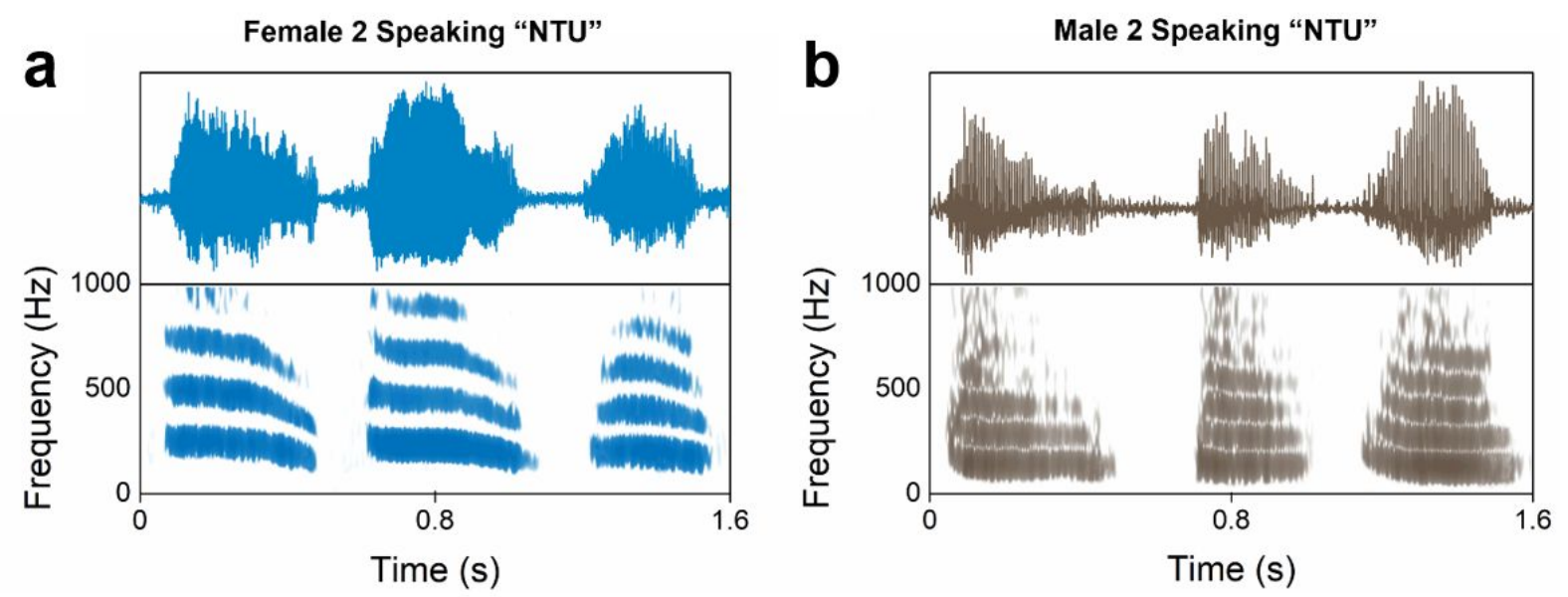

Figure S21. Acoustic signal waveforms and spectrograms of the phonation of "NTU" by speaker female 2 (a) and male 2 (b).

Table S1. A comparison of anti-interference performance among different skin-attached acoustic sensors.

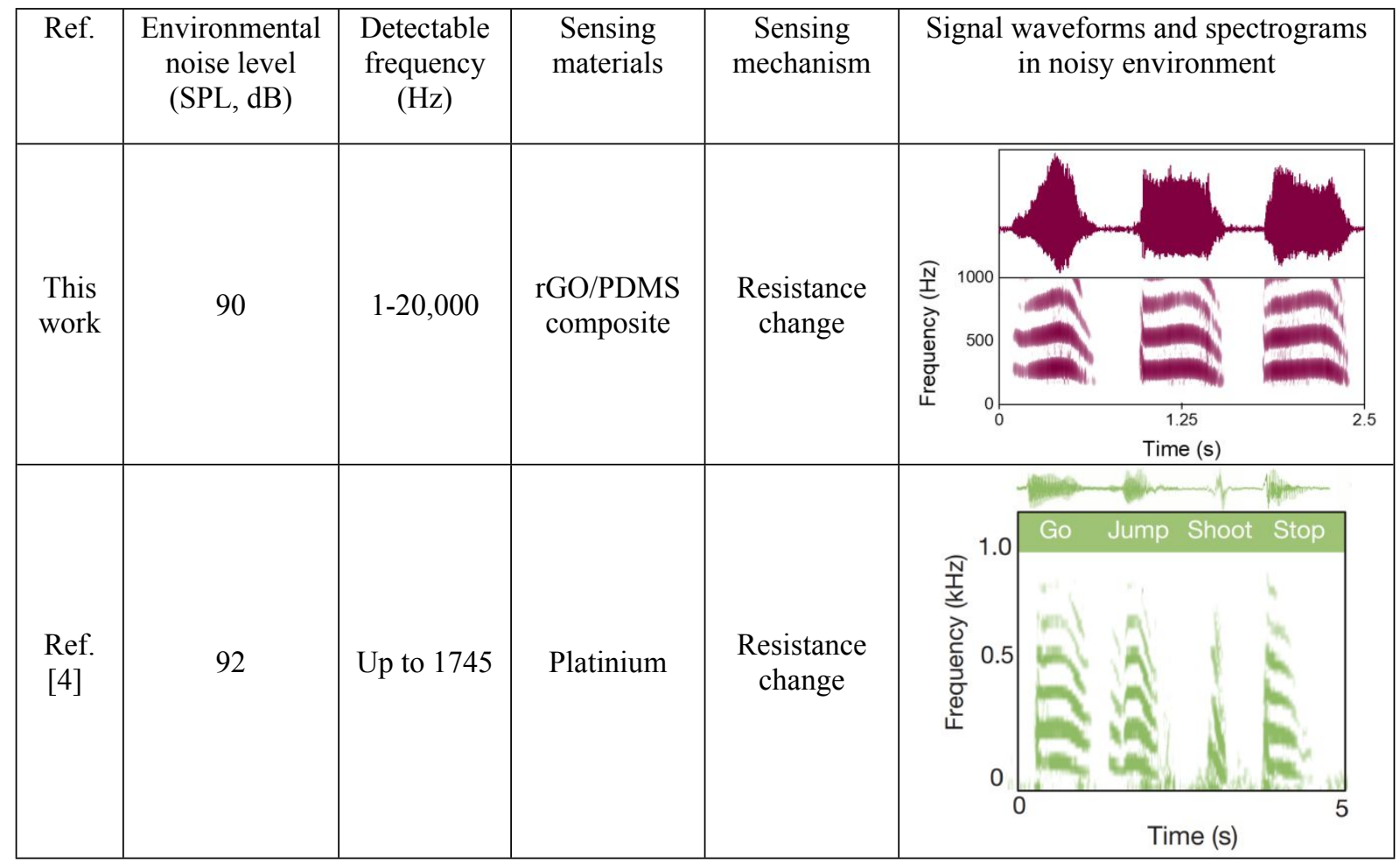




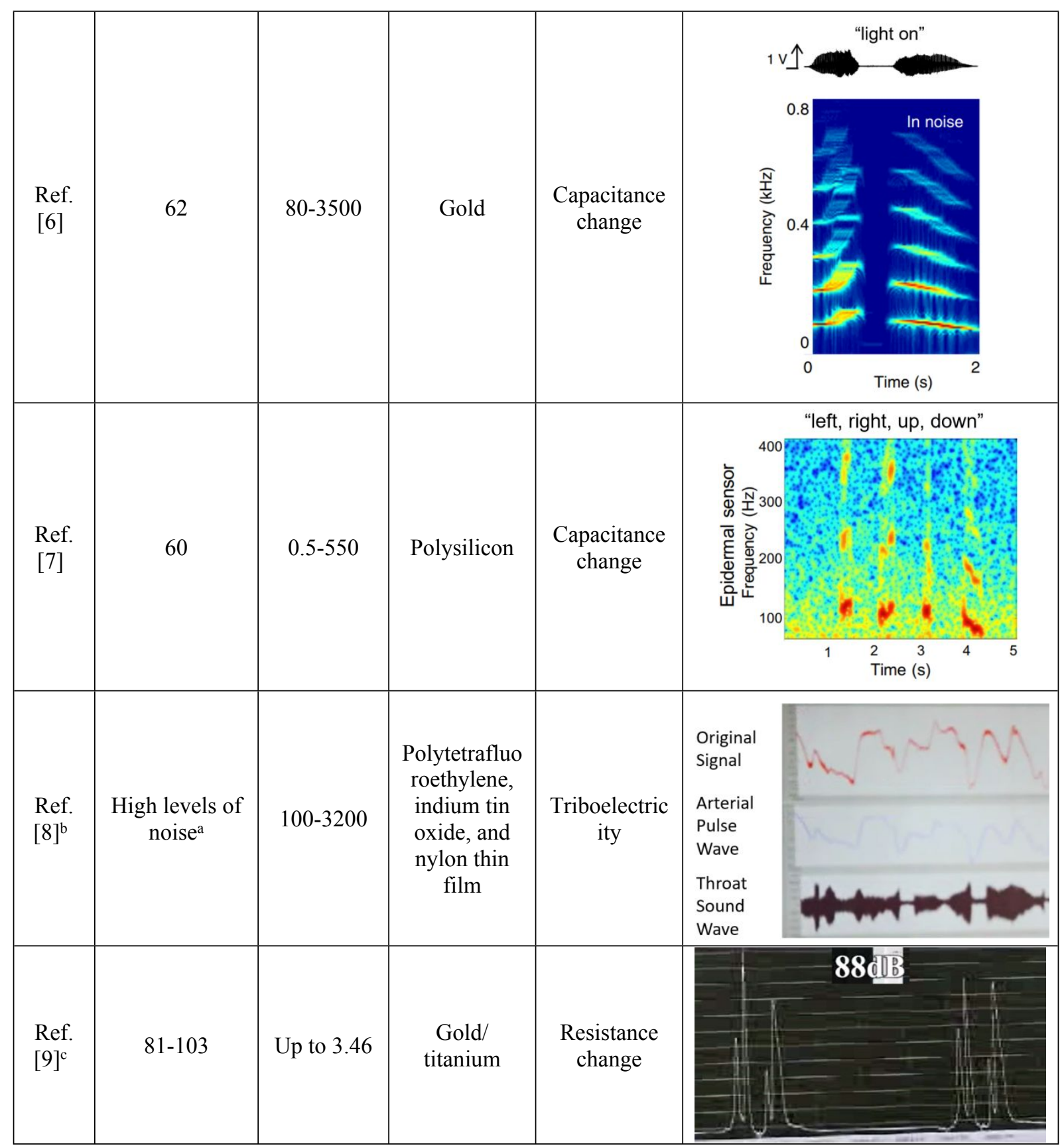

a: The authors do not mention the exact noise level. They describe the noise condition as "high levels of noise" in the paper.

b, c: The images were captured from supporting Movie S5 of Ref [8] and Movie S4 of Ref [9]. In the papers, the authors do not provide any official spectrogram or waveform recorded in noisy environment. 


\begin{tabular}{|ll|}
\hline SHG & : Second harmonic generation \\
MS & $:$ Mechanical shutter \\
HWP & $:$ Half-wave plate \\
PBS & $:$ Polarizing beam splitter \\
BD & $:$ Beam dump \\
$\mathrm{M}_{1}, \mathrm{M}_{2}$ & $:$ Mirrors \\
$\mathrm{GM}_{1}, \mathrm{GM}_{2}$ & : Galvano mirrors \\
\hline
\end{tabular}

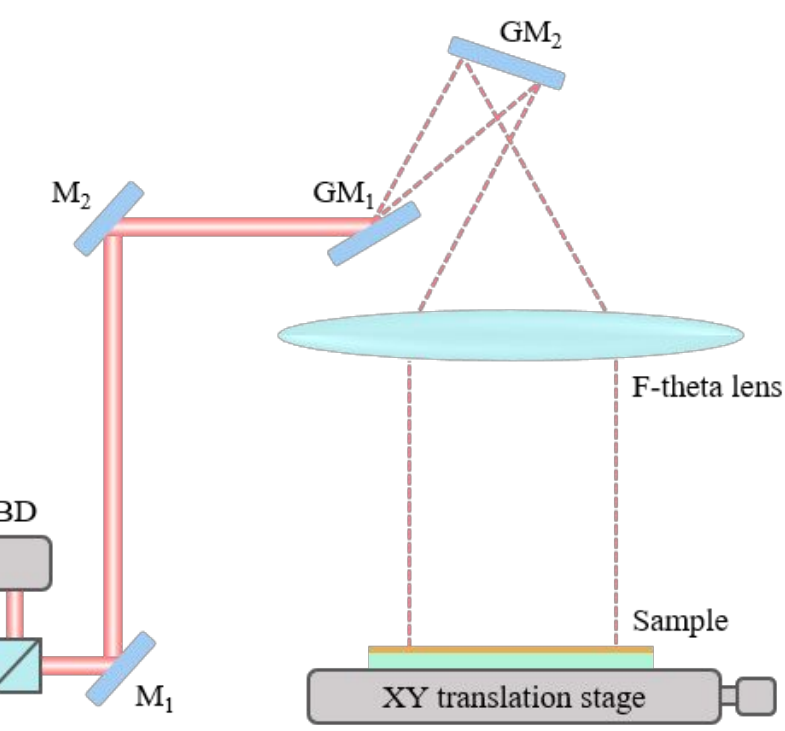

MS HWP PBS

Figure S22. Schematic of the FsLDW platform. 
Movie S1. Water repellency of the superhydrophobic rGO/PDMS composite film.

Movie S2. Water droplet impact on the superhydrophobic rGO/PDMS composite film.

Movie S3. Sliding of a water droplet on the superhydrophobic rGO/PDMS composite film.

Movie S4. Removal of contaminants on the rGO/PDMS composite film by pour water.

Movie S5. Water-proof capability of the rGO/PDMS composite strain sensor without any encapsulation.

Movie S6. Acoustic sensor attached on human neck for speech recognition of different letters and words.

Movie S7. Anti-interference speech recognition capability of the skin-attachable acoustic sensor when attached on human neck.

Movie S8. Demonstration of a smart voice-controlled security system based on the skinattachable acoustic sensor. 


\section{References}

1. Armyanov, S.; Stankova, N. E.; Atanasov, P. A.; Valova, E.; Kolev, K.; Georgieva, J.; Steenhaut, O.; Baert, K.; Hubin, A. XPS and $\mu$-Raman Study of Nanosecond-Laser Processing of Poly(Dimethylsiloxane) (PDMS). Nucl. Instrum. Methods Phys. Res. B 2015, 360, 30-35.

2. $\quad$ Song, H.; Zhang, J.; Chen, D.; Wang, K.; Niu, S.; Han, Z.; Ren, L. Superfast and HighSensitivity Printable Strain Sensors with Bioinspired Micron-Scale Cracks. Nanoscale 2017, 9, 1166-1173.

3. Wang, Z.; Volinsky, A. A.; Gallant, N. D. Crosslinking Effect on Polydimethylsiloxane Elastic Modulus Measured by Custom-Built Compression Instrument. J. Appl. Polym. 2014, 131,41050 .

4. Kang, D.; Pikhitsa, P. V.; Choi, Y. W.; Lee, C.; Shin, S. S.; Piao, L.; Park, B.; Suh, K. Y.; Kim, T. I.; Choi, M. Ultrasensitive Mechanical Crack-Based Sensor Inspired by the Spider Sensory System. Nature 2014, 516, 222-6.

5. Basu, A.; Roy, K.; Sharma, N.; Nandi, S.; Vaidhyanathan, R.; Rane, S.; Rode, C.; Ogale, S. CO2 Laser Direct Written MOF-Based Metal-Decorated and Heteroatom-Doped Porous Graphene for Flexible All-Solid-State Microsupercapacitor with Extremely High Cycling Stability. ACS Appl. Mater. Interfaces 2016, 8, 31841-31848.

6. Lee, S.; Kim, J.; Yun, I.; Bae, G. Y.; Kim, D.; Park, S.; Yi, I. M.; Moon, W.; Chung, Y.; Cho, K. An Ultrathin Conformable Vibration-Responsive Electronic Skin for Quantitative Vocal Recognition. Nat. Commun. 2019, 10, 2468.

7. $\quad$ Liu, Y.; Norton, J. J.; Qazi, R.; Zou, Z.; Ammann, K. R.; Liu, H.; Yan, L.; Tran, P. L.; Jang, K.-I.; Lee, J. W. Epidermal Mechano-Acoustic Sensing Electronics for Cardiovascular Diagnostics and Human-Machine Interfaces. Sci. $A d v$. 2016, 2, e1601185.

8. $\quad$ Yang, J.; Chen, J.; Su, Y.; Jing, Q.; Li, Z.; Yi, F.; Wen, X.; Wang, Z.; Wang, Z. L. Eardrum-Inspired Active Sensors for Self-Powered Cardiovascular System Characterization and Throat-Attached Anti-Interference Voice Recognition. Adv. Mater. 2015, 27, 1316-26.

9. Wang, C.; Zhao, J.; Ma, C.; Sun, J.; Tian, L.; Li, X.; Li, F.; Han, X.; Liu, C.; Shen, C.; Dong, L.; Yang, J.; Pan, C. Detection of Non-Joint Areas Tiny Strain and Anti-Interference Voice Recognition by Micro-Cracked Metal Thin Film. Nano Energy 2017, 34, 578-585. 